LAWRENCE LIVERMORE N A T IO N A L LABORATORY
Generation of high-contrast millijoule pulses by optical parametric chirped-pulse amplification in periodically poled KTiOPO4

I. Jovanovic, N. Forget, C. G. Brown, C. A. Ebbers, C. Le Blanc, C. P. J. Barty

October 4, 2004

Optics Letters 
This document was prepared as an account of work sponsored by an agency of the United States Government. Neither the United States Government nor the University of California nor any of their employees, makes any warranty, express or implied, or assumes any legal liability or responsibility for the accuracy, completeness, or usefulness of any information, apparatus, product, or process disclosed, or represents that its use would not infringe privately owned rights. Reference herein to any specific commercial product, process, or service by trade name, trademark, manufacturer, or otherwise, does not necessarily constitute or imply its endorsement, recommendation, or favoring by the United States Government or the University of California. The views and opinions of authors expressed herein do not necessarily state or reflect those of the United States Government or the University of California, and shall not be used for advertising or product endorsement purposes. 


\title{
Generation of high-contrast millijoule pulses by optical parametric chirped-pulse amplification in periodically poled $\mathrm{KTiOPO}_{4}$
}

\author{
Igor Jovanovic $^{\mathrm{a}}$, Nicolas Forget ${ }^{\mathrm{b}}$, Curtis G. Brown ${ }^{\mathrm{a}}$, Christopher A. Ebbers ${ }^{\mathrm{a}}$, \\ Catherine Le Blanc ${ }^{b}$, and C. P. J. Barty ${ }^{a}$ \\ ${ }^{\mathrm{a}}$ Lawrence Livermore National Laboratory, Mail Code L-470, \\ 7000 East Avenue, Livermore, California 94550 \\ baboratoire pour l'Utilisation des Lasers Intenses, École Polytechnique \\ Route de Saclay, 91128 Palaiseau cedex, France
}

\begin{abstract}
A new high-contrast, high-gain optical parametric chirped-pulse amplifier (OPCPA) architecture is demonstrated in periodically poled $\mathrm{KTiOPO}_{4}(\mathrm{PPKTP}$. This architecture overcomes parametric fluorescence contrast limitations of OPCPA in periodically poled materials. The scheme is based on two passes of a single, relay imaged, pump pulse and a free-propagating signal pulse through a $1.5 \times 5 \times 7.5 \mathrm{~mm}^{3}$ PPKTP crystal. The output energy of $1.2 \mathrm{~mJ}$ is generated at a center wavelength of 1053 $\mathrm{nm}$ utilizing $24 \mathrm{~mJ}$ of pump energy. Prepulse contrast level of $>3 \times 10^{7}$ has been measured with $>10^{6}$ saturated gain in the amplifier. Amplified pulses were compressed to $200 \mathrm{fs}$. This simple and versatile concept requires only a modest pump energy from a commercial pump laser and represents a possible high-contrast front end for high-energy Nd:glass-based petawatt-class lasers.
\end{abstract}

OCIS codes: (190.4970) Optical parametric amplifiers and optical parametric oscillators; (320.7090) Ultrafast lasers. 
In this paper we present for the first time an experimental demonstration and characterization of a double-pass, broad-bandwidth quasi-phase-matched (QPM) optical parametric chirped-pulse amplifier (OPCPA) in a single periodically poled $\mathrm{KTiOPO}_{4}$ (PPKTP) crystal that utilizes a single 24-mJ pump pulse from a commercial pump laser and produces $1.2 \mathrm{~mJ}$ of amplified signal energy, exhibits high gain $\left(>3 \times 10^{6}\right)$, good overall conversion efficiency $(5 \%)$, high prepulse contrast $\left(>3 \times 10^{7}\right)$, high energy stability (3\% rms), and excellent beam quality. To our knowledge, this is the greatest energy to date produced in QPM OPCPA.

Generation and amplification of short optical pulses requires gain media which can support broad spectral bandwidth. Other desirable properties of short-pulse amplifiers include high gain, high prepulse contrast, good thermal properties, low B-integral, and good beam quality. OPCPA $^{1,2}$ has been recently introduced as an amplification technology which exhibits these favorable properties. ${ }^{3,4}$ Additionally, OPCPA can exhibit high energy stability and high pump conversion efficiency when utilizing either optimized pump lasers ${ }^{5}$ or when used in a hybrid chirped-pulse amplification scheme. ${ }^{6}$

QPM OPCPA in periodically poled materials such as periodically poled $\mathrm{LiNbO}_{3}$ $(\text { PPLN })^{7}$ and PPKTP ${ }^{8,9}$ has been shown to exhibit additional advantages over the OPCPA in bulk nonlinear crystals. Those advantages include the access to the greater material nonlinear coefficient, resulting in ultra-high gain with low pump peak power, propagation along one of the crystal principal axes resulting in absence of birefringent walk-off, low angular sensitivity, improved beam quality.

An obstacle to simple high-gain amplification of optical pulses from $\mathrm{nJ}$ to $\mathrm{mJ}$ energies using periodically poled crystals has been the high level of parasitic parametric 
fluorescence (PF) when a single-pass, high-gain $\left(>10^{6}\right)$ amplification is sought. PF in QPM is increased when compared to PF in critical phase matching as a result of broader angular acceptance of the nonlinear conversion process. PF reduces prepulse contrast and conversion efficiency by competing with the signal pulse for pump pulse energy. Past approaches to QPM OPCPA were thus limited to small (tens of $\mu \mathrm{J}$ ) pulse energies. ${ }^{8,9}$ Optical parametric amplification of a narrowband signal pulse in PPKTP utilizing two pump beams has also been demonstrated at a mJ-level ${ }^{10}$, but the conversion efficiency has been limited by low energy extraction of pump pulse in the first pass of amplification. Additionally, narrow spectral bandwidth was the result of operation far from signatidler degeneracy.

Our experimental setup is presented in Fig. 1. The seed pulse centered at $1053 \mathrm{~nm}$ and with $6 \mathrm{~nm}$ FWHM bandwidth is generated by a mode-locked Yb:glass oscillator (High Q Laser IC-1053-200) operating at $100 \mathrm{MHz}$. The oscillator produces 2-nJ pulses which are introduced into a pulse stretcher of a chirped-pulse amplification system. The pulse stretcher utilizes a $1480 \mathrm{~mm}^{-1}$ diffraction grating and a plano-convex lens, resulting in spherical and chromatic aberrations which have previously limited recompression to $\sim 200$ fs. Stretching results in 800-pJ pulses with 1.2-ns FWHM duration, which are subsequently introduced into a Pockels cell pulse selector. While the use of Pockels cell is not required for normal OPCPA operation, its utilization helps the system characterization by removing a significant fraction of the unamplified seed pulse train.

Pump pulses are generated by a commercial, injection-seeded Nd:YAG laser (Continuum Powerlite Plus), producing 532-nm pulses with 5.7-ns FWHM pulse duration in the pulse center with a spatiotemporal shape previously measured from an unstable 
resonator. ${ }^{4}$ The repetition rate of the pump haser is $10 \mathrm{~Hz}$ and its pulses are synchronized with the oscillator pulses with a characteristic pulse-to-pulse jitter of $0.5 \mathrm{~ns}$ rms. A small amount of the available pump energy is split from the pump laser and a circular aperture of $\sim 7.5 \mathrm{~mm}$ diameter is inserted in the beam. The aperture is relay imaged using a 5:1 beam relay telescope onto a $1.5 \times 5 \times 7.5 \mathrm{~mm}^{3}$ PPKTP crystal (Raicol). The PPKTP crystal is periodically poled for a first-order, type-0 $1053 \mathrm{~nm}+1074 \mathrm{~nm}=532 \mathrm{~nm}$ process and both its input and output surfaces are antireflection coated for $532 \mathrm{~nm} / 1053 \mathrm{~nm} / 1075 \mathrm{~nm}$. The crystal is wedged $3^{\circ}$ on its back surface to prevent parasitic oscillation.

Seed and signal pulses are combined on a dichroic beamsplitter prior to injection into the PPKTP crystal with a small noncollinear angle of $<0.5^{\circ}$. Seed pulses overfill the crystal aperture, so that $\sim 50 \%$ of the available seed energy is introduced into the crystal. Optimal phase matching is achieved at the crystal temperature of $32^{\circ} \mathrm{C}$. After the first pass of amplification, pump is separated from signal and idler using the second dichroic beamsplitter and is fully relay imaged onto a roof mirror retro reflector. The roof mirror retro reflector displaces the pump beam laterally by $\sim 2 \mathrm{~mm}$ on the second (reverse) pass through the PPKTP crystal. Signal and idler pulses freely propagate without relay imaging to the second flat-mirror retroreflector. Idler pulses are separated from signal pulses using a knife edge $(\mathrm{K})$. The distance to the signal mirror retroreflector is nearly equal to the relay distance of the pump beam, allowing good temporal matching in two passes. Adjustments can be made in the length of the signal beam return path, allowing temporal multiplexing for longer pump pulses.

The fundamental advantage of the double-pass amplification scheme over the single-pass amplification scheme is in the reduction of PF through propagation of the 
signal/idler pulse over extended distances, thus allowing angularly divergent PF to be separated from the main signal/idler pulse prior to second-pass amplification. This property allows high-energy, high-gain amplification with a fluorescence level orders-ofmagnitude lower than previously. The removed fraction of PF can be increased by spatial filtering of the signal between two amplification passes.

We have obtained $1.2 \mathrm{~mJ}$ of amplified signal energy using $24 \mathrm{~mJ}$ of pump energy. If the measured spatiotemporal evolution of the pump pulse is taken into account, $4.8 \mathrm{~mJ}$ of the pump energy is inside the \pm 1.5 -ns sharp-edge temporal window defined by the stretcher bandpass. The conversion efficiency to signal and idler in this window is $\sim 50 \%$ with a gain of $3 \times 10^{6}$. Amplified spectrum exhibits spectral broadening to $12 \mathrm{~nm}$ FWHM (Fig. 2), which has been previously observed in saturated OPCPA. Pump and amplified signal near-field beam profiles are shown in Fig. 3. In Fig. 4 we show amplified signal free propagation and far field beam profiles. Excellent beam quality of the amplified signal after free propagation is the result of the diffraction of the higher order spatial modes, leaving only the low-order, high-beam-quality contributing spatial modes in the amplified pulse. It is thus expected that the overall conversion efficiency is greater than $50 \%$, since a fraction of the amplified energy is diffracted prior to measurement.

Amplified signal pulse has been recompressed and its intensity autocorrelation is shown in Fig. 5. The deconvolved pulse width of $206 \mathrm{fs}$ is calculated from the measured spectrum and intensity autocorrelation. Transform-limited recompression to $147 \mathrm{fs}$ would result from the measured amplified spectrum and is not fully achieved, which is attributed to spherical and chromatic aberrations in the pulse stretcher which contains and planoconvex lens. The aberrations in this system have been noted previously and have limited 
the recompression of unamplified pulses to 200 fs. Our measured compressed pulse width is therefore typical for this setup and not influenced by phase aberrations of OPCPA in PPKTP.

We have measured the prepulse and fluorescence contrast of the amplified signal pulse after recompression and determined to be $>3 \times 10^{7}$, limited by the experimental sensitivity of our measurement. The measured energy stability is $3 \% \mathrm{rms}$ and is greatly enhanced when local pump depletion occurs.

In conclusion, we have demonstrated for the first time multi-pass QPM OPCPA in PPKTP pumped by a commercial pump laser that produced record pulse energies of 1.2 $\mathrm{mJ}$, spectrally broadened from $6 \mathrm{~nm}$ to $12 \mathrm{~nm}$ and subsequently compressed to $206 \mathrm{fs}(1.4$ times their transform-limited pulse duration). Simple modification of the demonstrated scheme allows time-multiplexing for higher energy extraction in situations when pump pulse is much longer than the signal pulse. QPM OPCPA exhibits advantages over conventional OPCPA in bulk nonlinear materials. They include the access to the greater nonlinear coefficient resulting in reduced pump pulse energies, absence of birefringent walk-off resulting in higher conversion efficiencies, better beam quality, and relaxed requirements on pump pulse spatial profile from commercial pump lasers. QPM OPCPA also benefits from much broader ( 100 times) angular acceptance compared to bulk nonlinear crystals, resulting in noncritical alignment and stability, with possible very high conversion efficiencies from small, angularly divergent and multimode pump beams. Finally, double-pass QPM OPCPA overcomes the previous limitation of PF in high-gain amplification, enabling $>10^{6}$ gain from a single nonlinear crystal and a single pump pulse. 
This amplifier could be used in a high-gain section of a larger OPCPA system which has previously employed two or more crystals and higher pump energies, or a stand-alone, high-contrast preamplifier for a high-energy Nd:glass short-pulse laser. Future energy scaling of OPCPA in periodically poled materials is contingent upon improvements in manufacturing of thick ( $>2 \mathrm{~mm}$ ) periodically poled crystals of sufficient quality. While we have not observed any crystal degradation in $\sim 48$ hours of continuous operation at full energy, additional work is required to identify the preferred nonlinear material with greatest resistance to photorefraction and other detrimental processes leading to possible damage or degradation over extended operation.

This work was performed under the auspices of the U. S. Department of Energy by the University of California, Lawrence Livermore National Laboratory under Contract No. W-7405-Eng-48. 


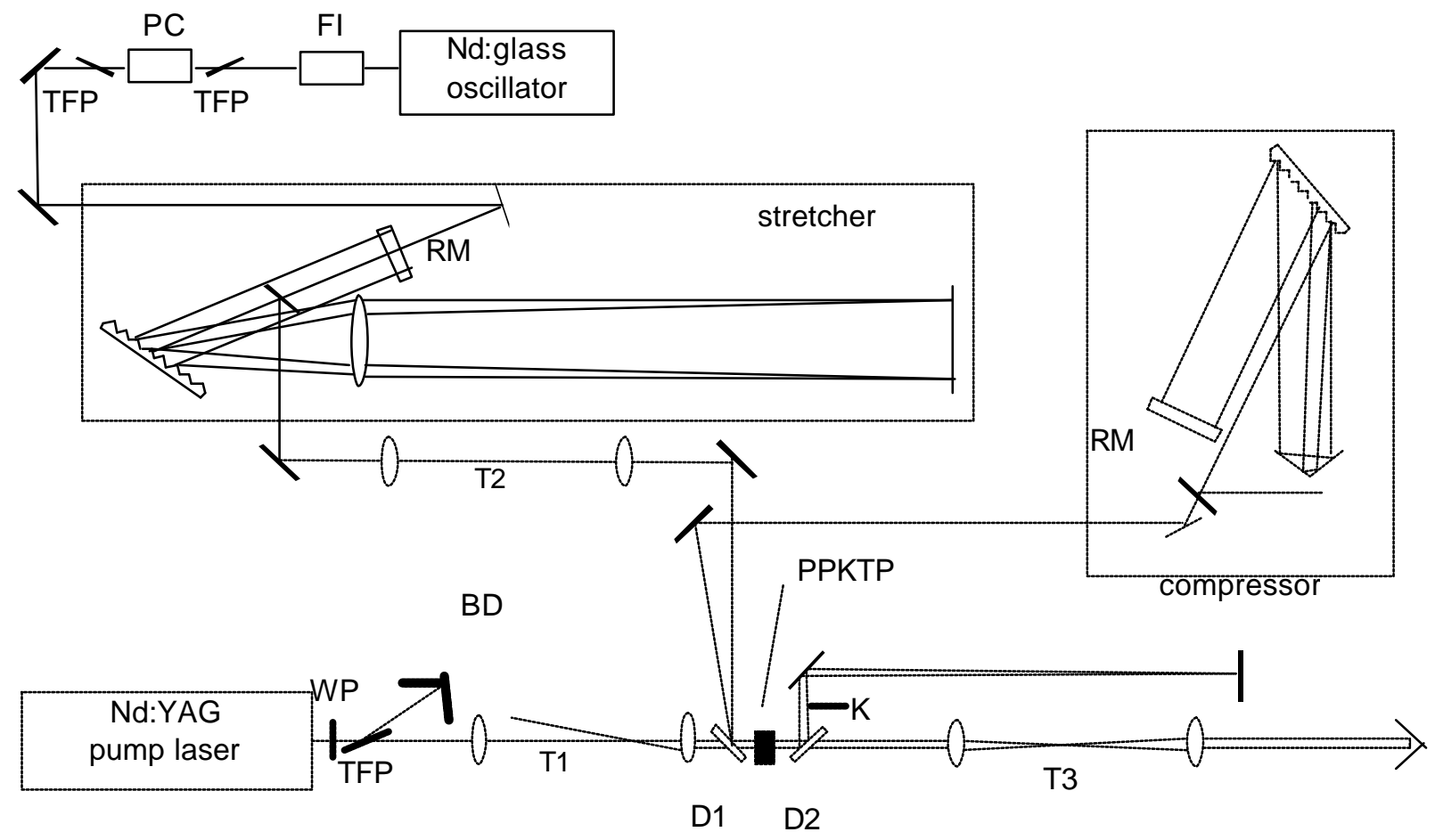

Figure. 1. Schematic for millijoule OPCPA in PPKTP. FI - Faraday isolator, PC Pockels cell, TFP - thin film polarizer, RM - roof mirror, T - telescope, WP - waveplate, D - dichroic beamsplitter, BD - beam dump, K-knife edge 


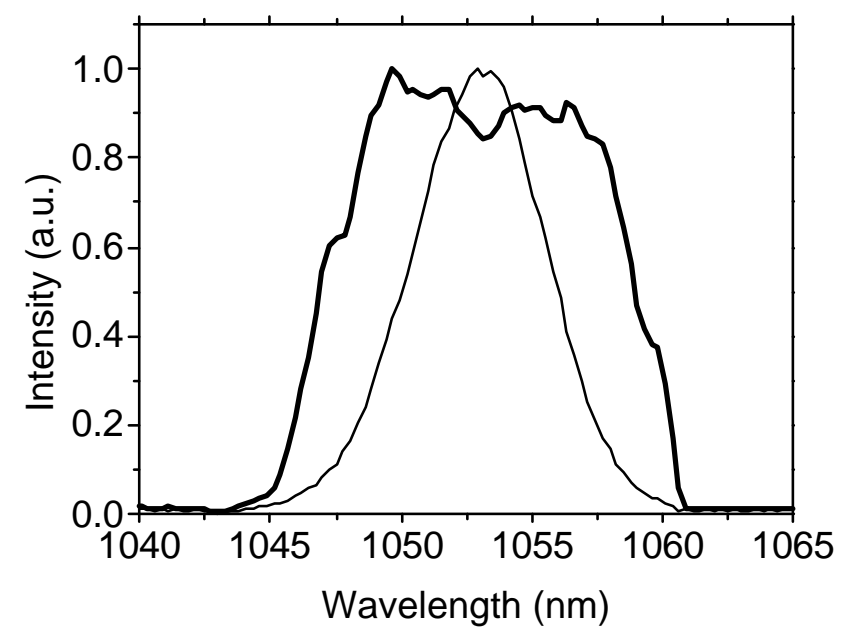

Figure 2. Oscillator (6 nm FWHM, thin line) and amplified signal spectra (12 nm FWHM, thick line) 


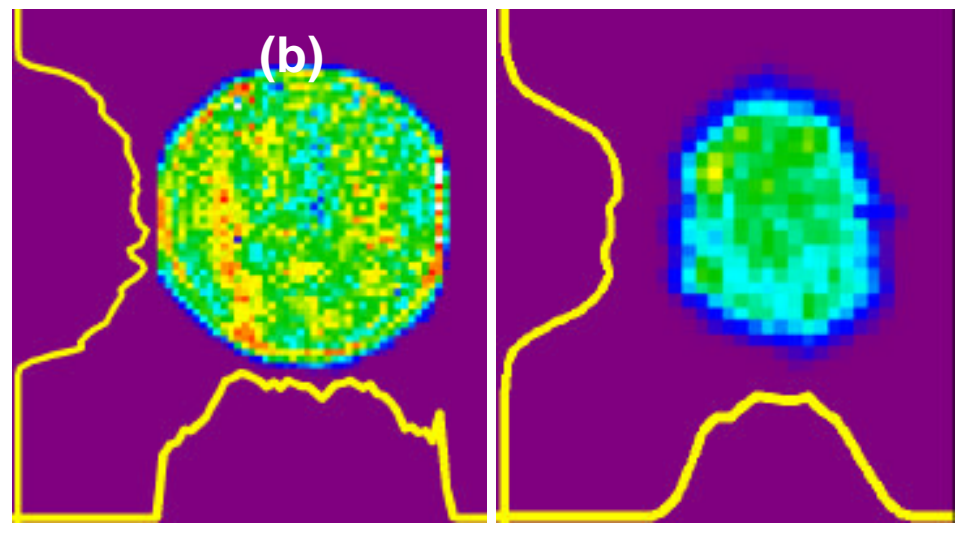

Figure 3. (a) Pump and (b) amplified signal (second pass) near field beam profiles 


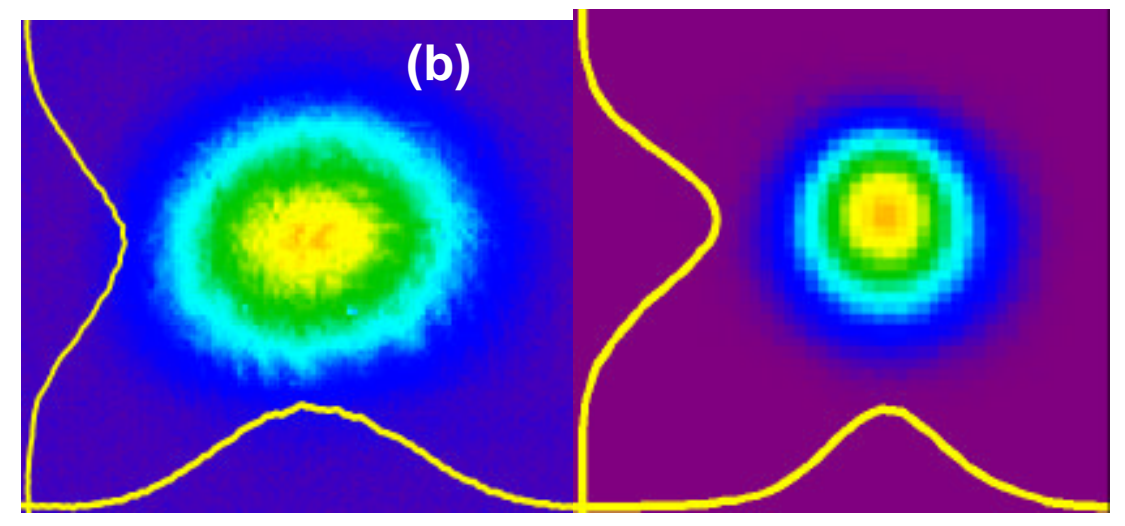

Figure 4. Amplified signal (a) free propagation and (b) far-field beam profiles 


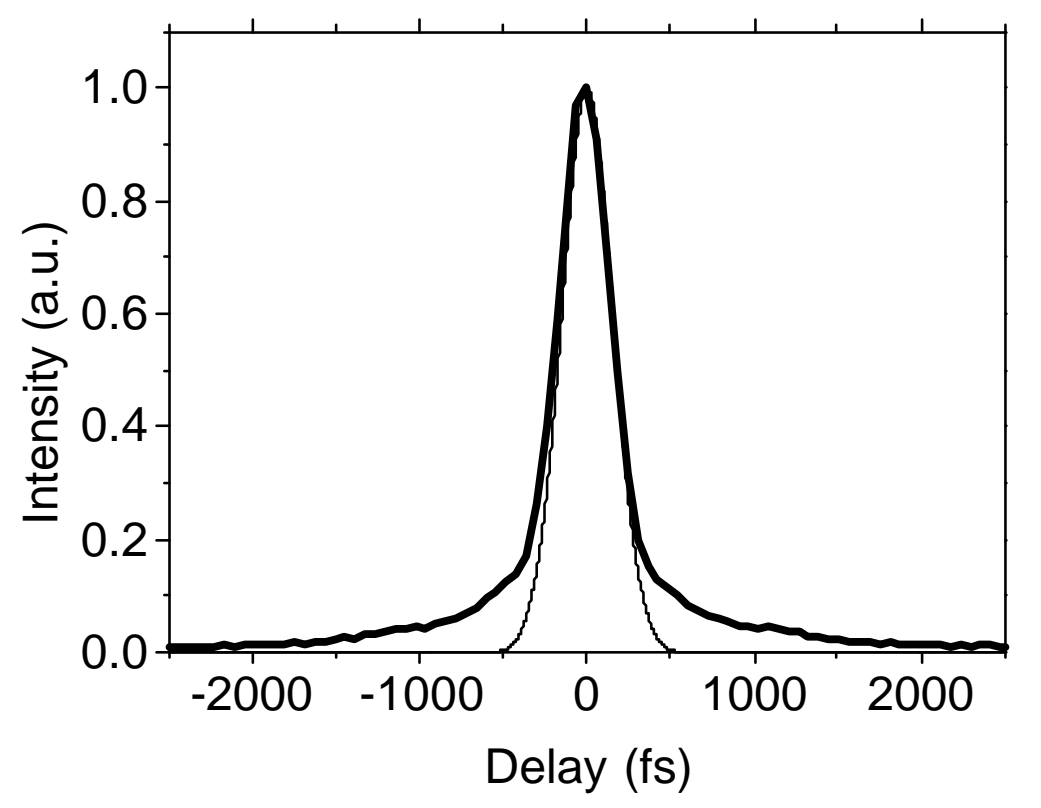

Figure 5. Experimental autocorrelation trace of the recompressed pulse (thick line; 282 fs FWHM; deconvolved pulse width 147 fs FWHM) and calculated transform-limited autocorrelation (thin line; 350 fs FWHM; deconvolved pulse width 206 fs FWHM). The recompressed pulse duration is 1.4 times the Fourier transform limit, resulting from spherical and chromatic aberrations in the pulse stretcher. 


\section{LIST OF REFERENCES (WITH FULL TITLES)}

1. A. Dubietis, G. Jonusauskas, and A. Piskarskas, ““'Powerful femtosecond pulse generation by chirped and stretched pulse parametric amplification in BBO crystal," Opt. Comm. 88, 437-440 (1992).

2. I. N. Ross, P. Matousek, M. Towrie, A. J. Langley, and J. L. Collier, “The prospects for ultrashort pulse duration and ultrahigh intensity using optical parametric chirped pulse amplifiers," Opt. Commun. 144, 125 (1997).

3. I. N. Ross, J. L. Collier, P. Matousek, C. N. Danson, D. Neely, R. M. Allott, D. A. Pepler, C. Hernandez-Gomez, and K. Osvay, "Generation of terawatt pulses by use of optical parametric chirped pulse amplification,” Appl. Opt. 39, 2422 (2000).

4. I. Jovanovic, B. J. Comaskey, C. A. Ebbers, R. A. Bonner, D. M. Pennington, and E. C. Morse, "Optical parametric chirped pulse amplifier as an alternative to Ti:sapphire egenerative amplifiers" 41, 2923 (2002).

5. L. J. Waxer, V. Bagnoud, I. A. Begishev, M. J. Guardalben, J. Puth, and J. D. Zuegel, "High-conversion-efficiency optical parametric chirped-pulse amplification system using spatiotemporally shaped pump pulses," Opt. Lett. 28, 1245 (2003).

6. I. Jovanovic, C. A. Ebbers, and C. P. J. Barty, “ Hybrid chirped-pulse amplification,” Opt. Lett. 27, 1622 (2002).

7. A. Galvanauskas, A. Hariharan, D. Harter, M. A. Arbore, and M. M. Fejer, "Highenergy femtosecond pulse amplification in a quasi-phase-matched parametric amplifier,” Opt. Lett. 23, 210 (1998).

8. F. Rotermund, V. Petrov, F. Noack, V. Pasiskevicius, J. Hellström, F. Laurell, H. Hundertmark, P. Adel, and C. Fallnich, “Compact all-Diode-Pumped Ferntosecond Laser 
Source based on Chirped Pulse Optical Parametric Amplification in Periodically Poled $\mathrm{KTiOPO}_{4}$," Electron. Lett. 38, 561 (2002).

9. I. Jovanovic, J. R. Schmidt, and C. A. Ebbers, "Optical parametric chirped-pulse amplification in periodically poled $\mathrm{KTiOPO}_{4}$ at $1053 \mathrm{~nm}$," Appl. Phys. Lett. 83, 4125 (2003).

10. A. Fragemann, V. Pasiskevicius, G. Karlsson, and F. Laurell, "High-peak power nanosecond optical parametric amplifier with periodically poled KTP,” Opt. Exp. 11, 1297 (2003). 


\section{LIST OF REFERENCES (WITHOUT TITLES)}

1. A. Dubietis, G. Jonusauskas, and A. Piskarskas, Opt. Comm. 88, 437-440 (1992).

2. I. N. Ross, P. Matousek, M. Towrie, A. J. Langley, and J. L. Collier, Opt. Commun. 144, 125 (1997).

3. I. N. Ross, J. L. Collier, P. Matousek, C. N. Danson, D. Neely, R. M. Allott, D. A. Pepler, C. Hernandez-Gomez, and K. Osvay, Appl. Opt. 39, 2422 (2000).

4. I. Jovanovic, B. J. Comaskey, C. A. Ebbers, R. A. Bonner, D. M. Pennington, and E. C. Morse, 41, 2923 (2002).

5. L. J. Waxer, V. Bagnoud, I. A. Begishev, M. J. Guardalben, J. Puth, and J. D. Zuegel, Opt. Lett. 28, 1245 (2003).

6. I. Jovanovic, C. A. Ebbers, and C. P. J. Barty, Opt. Lett. 27, 1622 (2002).

7. A. Galvanauskas, A. Hariharan, D. Harter, M. A. Arbore, and M. M. Fejer, Opt. Lett. 23, 210 (1998).

8. F. Rotermund, V. Petrov, F. Noack, V. Pasiskevicius, J. Hellström, F. Laurell, H. Hundertmark, P. Adel, and C. Fallnich, Electron. Lett. 38, 561 (2002).

9. I. Jovanovic, J. R. Schmidt, and C. A. Ebbers, Appl. Phys. Lett. 83, 4125 (2003). 10. A. Fragemann, V. Pasiskevicius, G. Karlsson, and F. Laurell, Opt. Exp. 11, 1297 (2003). 


\section{LIST OF FIGURES}

Figure 1. Schematic for millijoule OPCPA in PPKTP. FI - Faraday isolator, PC Pockels cell, TFP - thin film polarizer, RM - roof mirror, T - telescope, WP - waveplate, D - dichroic beamsplitter, BD - beam dump, K-knife edge

Figure 2. Oscillator (6 nm FWHM, thin line) and amplified signal spectra (12 nm

FWHM, thick line)

Figure 3. (a) Pump and (b) amplified signal (second pass) near field beam profiles

Figure 4. Amplified signal (a) free propagation and (b) far-field beam profiles

Figure 5. Experimental autocorrelation trace of the recompressed pulse (thick line; 282

fs FWHM; deconvolved pulse width 147 fs FWHM) and calculated transform-limited autocorrelation (thin line; 350 fs FWHM; deconvolved pulse width 206 fs FWHM). The recompressed pulse duration is 1.4 times the Fourier transform limit. 